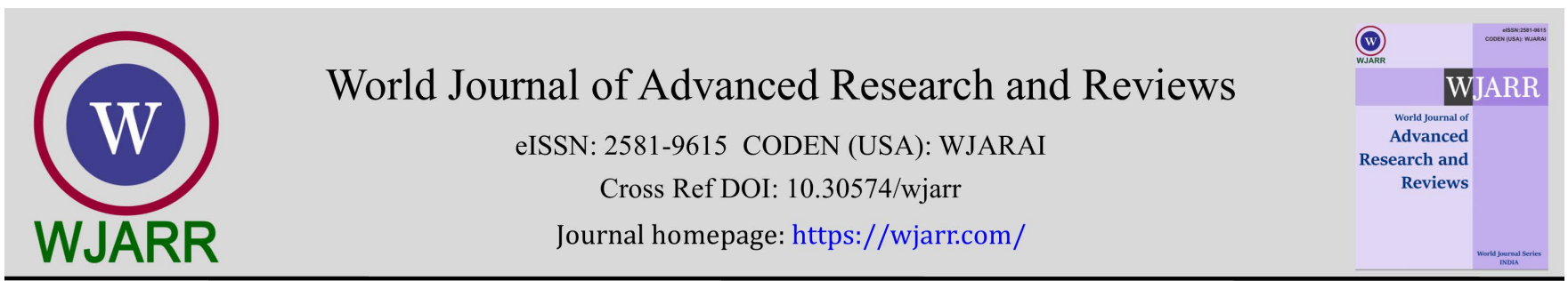

(RESEARCH ARTiClE)

\title{
The psychological effect of the COVID 19 pandemic on the staff of the Mohammed VI University Hospital in Marrakech
}

\author{
Mohamed IJIM *, Salma AITBATAHAR and Lamyae AMRO \\ Pulmonology department, Arrazi hospital, Mohammed VI University Hospital, Marrakech, Morocco.
}

World Journal of Advanced Research and Reviews, 2021, 12(03), 181-186

Publication history: Received on 07 November 2021; revised on 10 December 2021; accepted on 12 December 2021

Article DOI: https://doi.org/10.30574/wjarr.2021.12.3.0685

\begin{abstract}
Background: Health professionals have undergone very high levels of workload and pressure since the COVID-19 epidemic. Purpose: This study aims to assess the psychological impact of COVID-19 on health professionals at the Mohammed VI University Hospital. From May 31 to June 22, 2020.

Methods: This is a cross-sectional study carried out among professionals of the Mohammed VI University Hospital in Marrakech using a questionnaire measuring the psychological impacts; the event impact scale - revised (IES-R), An exploratory and bivariate factor analysis was carried out to exploit the results.

Results; 117 people answered the questionnaire, $66.67 \%$ were women, the average age was 30 years with extremes ranging from 25 to 60 years, $46.15 \%$ were doctors, $46.15 \%$ were nurses, $25.65 \%$ of participants lived alone, while $74.35 \%$ with families, after the start of the epidemic, 51 people lived in one of the hotels dedicated to housing and confinement. $28.2 \%$ or 33 people had declared transport problems. Of the participants in the study, 53.84\% presented with insomnia, $12.82 \%$ (15) described aggression, and somatization was presented by 9 people (7.69\%), nightmares by 9 others $(7.69 \%)$ and intense fear by 3 people. $7.7 \%$ or 9 people who have already consulted the psychiatric listening cell during this period. The IES-R score is calculated at 41.94 or in favor of a post-traumatic stress disorder prevalent in this sample, with a score of avoidance at 12.76, intrusion of memories at 18.58 and hyperactivity at 10.58 .
\end{abstract}

Conclusion: unwanted psychological impacts are prevalent among our healthcare professionals.

Keywords: Coronavirus; Depression; Anxiety; Health professionals; Psychological

\section{Introduction}

There is a broad consensus that the outbreak of an infectious disease is often linked to psychological impacts [1]. Containment measures, including mandatory quarantine or distancing, especially if it is prolonged, may increase the risk of psychological disorders, such as depression, anxiety, post-traumatic stress [2].

Compared to the general population, health workers are more likely to experience a wide range of negative psychological impacts following an emergency or disaster.

Severe emotional stress had been reported during or after the outbreak of infectious diseases among healthcare professionals in previous studies, including the 2003 Severe Acute Respiratory Syndrome (SARS) epidemic [3], Ebola virus disease in 2014 and Middle East respiratory syndrome epidemic (MERS) in 2015 [4,5].

\footnotetext{
* Corresponding author: Mohamed IJIM

Pulmonology department, Arrazi hospital, Mohammed VI University Hospital, Marrakech, Morocco.

Copyright $(2021$ Author(s) retain the copyright of this article. This article is published under the terms of the Creative Commons Attribution Liscense 4.0.
} 
Health workers have been shown to experience high levels of stress, anxiety, depression and post-traumatic stress during or even after the onset of infectious diseases [6].

Adverse psychological outcomes in healthcare workers are usually determined by a variety of factors during an infectious disease outbreak with a high death rate, including uncertain length of quarantine, inadequate medical supplies, fears of infection, stigma and discrimination, etc. [2, 7].

Meanwhile, the support they got from others and the coping strategies they adopted during the event had been reported to be associated with their psychological state during the disease outbreak [5]. Less support and more negative coping strategies have been shown to be common predictors of acute and chronic PTS and other mental health problems [8,9].

By understanding the psychological consequences caused by an epidemic in healthcare workers and studying the mechanism below, effective intervention and treatment can be developed and provided to this population, to improve their psychological well-being.

The present study aimed to investigate the presence of an adverse psychological outcome experienced by healthcare professionals during the COVID-19 epidemic and assess the associated factors,

\section{Methods}

This study was a quantitative and descriptive survey using the snowball sampling strategy, including doctors, nurses, medical technicians, and non-medical staff working in hospitals. Study participants were recruited from hospitals in the Mohammed VI University Center in Marrakech. The time span of the study was 22 days between May 31 to June 21, 2020. A total of 117 people voluntarily participated and completed questionnaires anonymously online. Thus the consent of the participants was obtained.

The survey questions included Sociodemographic variables (gender, age, marital status and occupation), psychiatric health status, confinement and working conditions during this period, Impact of Event Scale-Revised (IES- R).

The Impact of Event Scale (IES-R) was used to assess subjective stress caused by traumatic events, and it includes 22 items and consists of three subscales of symptoms of post-traumatic stress disorder (PTSD), including intrusion, hyperarousal and avoidance [10]. The IES-R in French version was used in our study.

The PTSD total score were converted to dichotomous variables (presence of PTSD symptoms (IES-R $\geq 22$ ) and no PTSD symptoms). The group comparisons of categorical variables were carried out with chi-square tests, and continuous variables were analyzed with Student's t-test. For univariate analysis of PTSD symptoms, the chi-square test was used for categorical variables. The count and frequency were presented.

Linear regressions were used to calculate bivariate associations between socio-demographic characteristics, variables related to the COVID-19 outbreak and psychological outcomes, for detecting risk factors for PTSD symptoms.

A two-sided $\mathrm{p}<0.05$ was identified as statistically significant, EPI INF0 7.2.4.0 software for windows was used for statistical analysis.

\section{Results}

117 people responded to the questionnaire, $66.67 \%$ were women, the average age was 30 years with extremes ranging from 25 to 60 years, of which $97 \%$ were under 40 years old, $53.85 \%$ were married, $46.15 \%$ were doctors, $46.15 \%$ were nurses, 15 people or $12.82 \%$ had already had psychiatric illnesses (depression: 9, anxiety: 3, panic disorder; 3), 25.65\% of the participants lived alone, while $74.35 \%$ with family, after the at the start of the epidemic, 51 people lived in one of the hotels dedicated to housing and confinement, the reception within these housing establishments was deemed satisfactory by $64.70 \%$ of the housed, and also the calm and security by the same percentage, while the quality of the food was deemed unsatisfied by $76.47 \%$ of the residents.

$28.2 \%$ or 33 people had declared transport problems between the accommodation and the hospital during the period of confinement. 
Among the participants in the study, 93 or $79.46 \%$ worked directly with patients confirmed to have covid 19 , the number of people who changed their post of assignment was 54 or $46.15 \%$, on average the number of working hours was $36 \mathrm{~h}$ per week per person,

The level of remuneration was judged unsatisfactory by $82.05 \%$ of the people, $51.28 \%$ judged the communication as unsatisfied, and 58.97 judged the availability of protective equipment as satisfactory.

The percentage of people who kept a regular physical activity was $15.39 \%, 10.26 \%$ of people who kept close physical contact with their families during this period of confinement, the degree of stress in general was considered very important by $66.67 \%$ of people,

Sixty three people or $53.84 \%$ presented with insomnia, $12.82 \%$ (15) described aggression, and somatization was presented by 9 people (7.69\%), nightmares by 9 others (7.69\%) and intense fear by 3 people. $7.7 \%$ or 9 people who have already consulted the psychiatric listening cell during this period.

The IES-R score is calculated at 41.94 or in favor of a post-traumatic stress disorder widespread in this sample, with a score of avoidance at 12.76 , intrusion of memories at 18.58 and hyperactivity at 10.58 (table 1 ).

Table 1 Association between the variables linked to the COVID-19 epidemic and the IES-R score $\geq 22$

\begin{tabular}{|l|c|c|c|c|c|}
\hline & \multicolumn{3}{|c|}{ IES-R $\geq 22$} & Mild-p exact & Fisher exact \\
\hline Gender & NO & YES & Total & 0.315 & 0.406 \\
\hline Female & 15 & 63 & 78 & & \\
\hline Row\% & $19.23 \%$ & $80.77 \%$ & $100.00 \%$ & & \\
\hline Male & 6 & 33 & 39 & & \\
\hline Row\% & $15.38 \%$ & $84.62 \%$ & $100.00 \%$ & & \\
\hline Marital status & & & & 0.374 & 0.464 \\
\hline Single & 9 & 45 & 54 & & \\
\hline Row\% & $16.67 \%$ & $83.33 \%$ & $100.00 \%$ & & \\
\hline Married & 12 & 51 & 63 & & \\
\hline Row\% & $19.05 \%$ & $80.95 \%$ & $100.00 \%$ & & \\
\hline CHANGE OF POSITION (job) & & & & 0.374 & 0.464 \\
\hline No & 12 & 51 & 63 & & \\
\hline Row\% & $19.05 \%$ & $80.95 \%$ & $100.00 \%$ & & \\
\hline Yes & 9 & 45 & 54 & & \\
\hline Row\% & $16.67 \%$ & $83.33 \%$ & $100.00 \%$ & & \\
\hline Psychiatric consultation & & & & 0.078 & \\
\hline No & 21 & 87 & 108 & & \\
\hline Row\% & $19.44 \%$ & $80.56 \%$ & $100.00 \%$ & & \\
\hline Yes & 0 & 9 & 9 & & \\
\hline Row\% & $0.00 \%$ & $100.00 \%$ & $100.00 \%$ & & \\
\hline Contact with family & & & & 0.255 & \\
\hline No & 18 & 87 & 105 & & \\
\hline Row\% & $17.14 \%$ & $82.86 \%$ & $100.00 \%$ & & \\
\hline Yes & 3 & 9 & 12 & & \\
\hline Row\% & $25.00 \%$ & $75.00 \%$ & $100.00 \%$ & & \\
\hline Habitat & & & & 0.089 & \\
\hline At home & $13.64 \%$ & $86.36 \%$ & $100.00 \%$ & & \\
\hline Row\% & 12 & 39 & 51 & & \\
\hline Hôtel & & & \\
\hline
\end{tabular}




\begin{tabular}{|l|c|c|c|c|c|}
\hline Row\% & $23.53 \%$ & $76.47 \%$ & $100.00 \%$ & & \\
\hline Alone or with family & & & & 0.363 & 0.463 \\
\hline With family & 15 & 72 & 87 & & \\
\hline Row\% & $17.24 \%$ & $82.76 \%$ & $100.00 \%$ & & \\
\hline Alone & 6 & 24 & 30 & & \\
\hline Row\% & $20.00 \%$ & $80.00 \%$ & $100.00 \%$ & & \\
\hline Sports activity & & & & 0.045 & 0.07 \\
\hline No & 15 & 84 & 99 & & \\
\hline Row\% & $15.15 \%$ & $84.85 \%$ & $100.00 \%$ & & \\
\hline Yes & 6 & 12 & 18 & & \\
\hline Row\% & $33.33 \%$ & $66.67 \%$ & $100.00 \%$ & & \\
\hline Working with covid + patients & & & & 0.232 & 0.326 \\
\hline No & 3 & 21 & 24 & & \\
\hline Row\% & $12.50 \%$ & $87.50 \%$ & $100.00 \%$ & & \\
\hline Yes & 18 & 75 & 93 & & \\
\hline Row\% & $19.35 \%$ & $80.65 \%$ & $100.00 \%$ & & \\
\hline
\end{tabular}

\section{Discussion}

The results of our study revealed a high prevalence of PTS among medical professionals during the COVID 19 virus outbreak. The rate was also outside the 10-27\% range of probable and clinical diagnoses of PTSD reported in the Ebola epidemic in 2014-2016 in the general population [7] and in the SARS epidemic among health workers in 2003 [11]. Post-traumatic stress disorder (PTSD) is a common psychiatric disorder manifested by symptoms of intrusion, overarousal and avoidance following a traumatic event [12]. According to previous studies, healthcare professionals are likely to develop unwanted psychological problems, such as depression and post-traumatic stress disorder as a result of their trauma experience $[13,14]$.

In the circumstances of an infectious disease outbreak, frontline health workers are always afraid of being infected or infecting others, especially when they have physical symptoms related to the infection $[2,13,15]$. During this time of containment health workers obviously became the population at high risk of transmitting the virus to who have close contact with them, and not surprisingly, were in the position of being stigmatized or left behind by others.

Stigma / distancing, fears of infection and the perceived high risk of their work; These are issues of great concern to healthcare professionals in the COVID-19 outbreak and other similar outbreaks, and have been shown to be associated with adverse psychological outcomes in this and other study. Other previous ones $[2,6,13,7]$.

Therefore, in addition to providing appropriate psychological counseling and accurate information targeting stigma against frontline health workers to mitigate their perceived threat, a more supportive social environment and more user-friendly mass media would be helpful for psychological health. Health workers during an infectiou disease epidemic.

In addition, the shortage of medical supplies among health professionals is always worth considering, it can lead to serious psychological consequences for them, even suicide.

Active coping strategies focused on problem solving can lead to an improvement in the relationship between the person and the environment and thus lead to a positive emotional response [16].

Our data suggest strategies that promote active coping styles and provide sufficient social support may help reduce the onset of unwanted psychological symptoms like depression, anxiety, and stress. This is consistent with previous studies that found active coping and social support to be the most important buffers of negative psychological health among healthcare workers $[17,18]$. 
Given the amount of stress experienced by medical personnel during the pandemic, it is important to provide them with personalized mental health support, such as observing changes in the trajectory of the post-pandemic mental health situation and setting up 'a nationwide psychological support group, in order to prevent the occurrence of generalized psychiatric disorders in this population.

Otherwise, it would be a long-term social and economic burden [19]. In addition, appropriate response measures must be adopted based on psychological assessment at every stage of the pandemic, including timely counseling and testing, development of positive coping strategies and creation of an environment more social friendly; environment and mass media network. This would apply to similar outbreaks in the future.

Our preliminary results can be supplemented by future studies targeting this population.

\section{Conclusion}

Unwanted psychological impacts are prevalent among healthcare professionals in Marrakech during the COVID-19 outbreak

Screening for adverse psychological effects and developing corresponding preventive measures would be beneficial in reducing the negative psychological outcomes of the COVID-19 pandemic among frontline soldiers

\section{Recommendations}

Unwanted psychological impacts are prevalent among healthcare professionals in Marrakech during the COVID-19 outbreak, the IES-R score is calculated at 41.94 or in favor of a post-traumatic stress disorder widespread in this sample, with a score of avoidance at 12.76, intrusion of memories at 18.58 and hyperactivity at 10.58.

\section{Compliance with ethical standards}

\section{Acknowledgments}

We would like to thank our masters, specialists, residents, and nurses from the pneumology department and all the staff of Marrakesh University Hospital for all the support they have provided during the preparation of this work.

\section{Authors' contributions}

Guarantor of integrity of entire study, all authors; study concepts/study design or data acquisition or data analysis/interpretation, all authors; manuscript drafting or manuscript revision for important intellectual content, all authors; approval of final version of submitted manuscript, all authors; agrees to ensure any questions related to the work are appropriately resolved, all authors; literature research, all authors; clinical studies, all authors.; statistical analysis, M.I.; and manuscript editing, all authors.

\section{Disclosure of conflict of interest}

The authors declare no conflict of interest.

\section{Statement of informed consent}

Permission to conduct the study was obtained from Department of Pneumology, ARRAZI Hospital, Mohamed VI University Hospital Center, Marrakesh. Informed consent was obtained from participants. All participants' information including raw data was be kept confidential during and after study period.

\section{References}

[1] El-Hage W, Hingray C, Lemogne C, Yrondi A, Brunault P, BienvenuT, Etain B, Paquet C, Gohier B, Bennabi D, Birmes P, Sauvaget A, Fakra E, Prieto N, Bulteau S, Vidailhet P, Jollant F, Camus V, Leboyer M, Krebs M-Odile, Aouizerate $\mathrm{B}$. Health professionals facing the coronavirus disease pandemic (COVID-19): what are the risks for their mental health ?. The Encephale. (2020).

[2] Brooks SK, Webster RK, Smith LE, Woodland L, Wessely S, Greenberg N, Rubin GJ. The psychological impact of quarantine and how to reduce it: rapid review of the evidence. Lancet. 2020; 395(10227): 912-920. 
[3] Tam CWC, Pang EPF, Lam LCW, Chiu HFK. Severe acute respiratory syndrome (SARS) in Hong Kong in 2003: Stress and psychological impact among frontline healthcare workers. Psychological Medicine. $2004 ; 34$ (7): 1197-1204.

[4] Lee SM, Kang WS, Cho AR, Kim T, Park JK. Psychological impact of the 2015 MERS outbreak on hospital workers and quarantined hemodialysis patients. Comprehensive Psychiatry. 2018; 87: 123-127.

[5] Raven J, Wurie H, Witter S. Health workers' experiences of coping with the Ebola epidemic in Sierra Leone's health system: a qualitative study. Bmc Health Services Research. 2018; 18.

[6] Lancee WJ, Maunder RG, Goldbloom DS, Study S. Prevalence of psychiatric disorders among Toronto hospital workers one to two years after the SARS outbreak. Psychiatric Services. 2008; 59(1): 91-95.

[7] Jalloh MF, Li WS, Bunnell RE, Ethier KA, O'Leary A, Hageman KM, Sengeh P, Jalloh MB, Morgan O, Hersey S et al. Impact of Ebola experiences and risk perceptions on mental health in Sierra Leone.July 2015. Bmj Global Health 2018; 3(2).

[8] Ahmadian A, Mirzaee J, Omidbeygi M, Holsboer-Trachsler E, Brand S. Differences in maladaptive schemas between patients suffering from chronic and acute posttraumatic stress disorder and healthy controls. Neuropsychiatric Disease and Treatment. 2015; 11: 1677-1684.

[9] Meli L, Birk J, Edmondson D, Bonanno GA. Trajectories of posttraumatic stress in patients with con'rmed and rule-out acute coronary syndrome. General Hospital Psychiatry. 2020; 62: 37-42.

[10] Thoresen S, Tambs K, Hussain A, Heir T, Johansen VA, Bisson JI. Brief measure of posttraumatic stress reactions: Impact of Event Scale-6. Social Psychiatry and Psychiatric Epidemiology. 2010; 45(3): 405-412.

[11] Wu P, Fang YY, Guan ZQ, Fan B, Kong JH, Yao ZL, Liu XH, Fuller CJ, Susser E, Lu J et al. The Psychological Impact of the SARS Epidemic on Hospital Employees in China: Exposure, Risk Perception, and Altruistic Acceptance of Risk. Canadian Journal of Psychiatry. 2009; 54 (5): 302-311.

[12] Astill Wright L, Sijbrandij M, Sinnerton R, Lewis C, Roberts NP, Bisson JI. Pharmacological prevention and early treatment of post-traumatic stress disorder and acute stress disorder: a systematic review and meta-analysis. Translational Psychiatry. 2019; 9(1): 334.

[13] Bai Y, Lin CC, Lin CY, Chen JY, Chue CM, Chou P. Survey of stress reactions among health care workers involved with the SARS outbreak. Psychiatric Services. 2004; 55(9): 1055-1057.

[14] Wu P, Liu XH, Fang YY, Fan B, Fuller CJ, Guan ZQ, Yao ZL, Kong JH, Lu J, Litvak IJ. Alcohol Abuse/Dependence Symptoms Among Hospital Employees Exposed to a SARS Outbreak. Alcohol and Alcoholism. 2008; 43(6): 706712.

[15] Maunder R, Hunter J, Vincent L, Bennett J, Peladeau N, Leszcz M, Sadavoy J, Verhaeghe LM, Steinberg R, Mazzulli T. The immediate psychological and occupational impact of the 2003 SARS outbreak in a teaching hospital. Canadian Medical Association Journal. 2003; 168(10): 1245-1251.

[16] Folkman S, Lazarus R: Folkman S, Lazarus RS. (1988) Coping as a mediator of emotion. J Pers Soc Psychol. 1988; 54: 466-475.

[17] Li XF, Guan LL, Chang H, Zhang B. Core Self-Evaluation and Burnout among Nurses: The Mediating Role of Coping Styles. Plos One. 2014; $9(12)$.

[18] Garrosa E, Rainho C, Moreno-Jimenez B, Monteiro MJ. The relationship between job stressors, hardy personality, coping resources and burnout in a sample of nurses: A correlational study at two time points. International Journal of Nursing Studies. 2010; 47(2): 205-215.

[19] Sani G, Janiri D, Di Nicola M, Janiri L, Ferretti S, Chieffo D. Mental health during and after the COVID- 19 emergency in Italy. Psychiatry Clin Neurosci. 2020; 74(6): 372. 